Recepción: 20 / 01 / 2017

Aceptación: 20 / 02 / 2017

Publicación: 15 / 03 / 2017

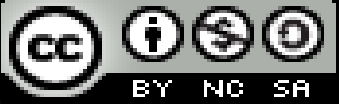

Ciencias Médicas

Articulo de investigación

\title{
Importancia del diagnóstico de apendicitis por ultrasonido
}

Importance of diagnosis of appendicitis by ultrasound

\section{Importância do diagnóstico de ultra-som de apendicite}

María Auxiliadora Calero-Zea ${ }^{\text {I }}$ maria.caleroz@ug.edu.ec

Blanca Elena Andrade-Burgos II

blanca.andradeb@ug.edu.ec

\author{
Amando Sánchez-Alcívar ${ }^{\mathrm{III}}$ \\ amando.sancheza@ug.edu.ec
}

Julio Ramón Villacrés-Pastor ${ }^{\text {IV }}$ julio.villacresp@ug.edu.ec

Correspondencia: maria.caleroz@ug.edu.ec

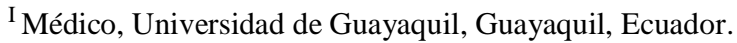

II Médico, Universidad de Guayaquil, Guayaquil, Ecuador.

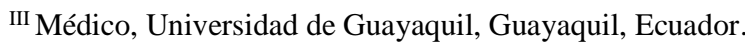

IV Médico, Universidad de Guayaquil, Guayaquil, Ecuador.
} 


\section{Resumen}

El dolor abdominal agudo es una enfermedad frecuente en niños. La apendicitis aguda es la más común indicación para la emergencia de cirugía abdominal en los niños. Abarcan el 80\% de las emergencias quirúrgicas. El diagnóstico de la apendicitis aguda ha sido tradicionalmente basado en los criterios clínicos.

La mayoría de los niños con apendicitis aguda presentan rasgos clínicos típicos y pasan por cirugía inmediata. Sin embargo, del $30-45 \%$ tienen rasgos clínicos atípicos, y un pronto diagnóstico es difícil en base de los hallazgos clínicos solamente, ya que los niños no son capaces de expresar sus molestias adecuadamente y los exámenes físicos pueden no ser precisos retardando la cirugía y las consecuencias pueden ser por lo contrario perforación, abscesos y peritonitis.

Esto además del peligro para el paciente puede resultar en hospitalización prolongada y un aumento en el riesgo de infertilidad en las pacientes mujeres. Así los persistentes desafíos son para establecer el diagnóstico temprano de la apendicitis atípica, suficiente como para poder prevenir la perforación y sus consecuencias.

Palabras clave: Apendicitis; ultrasonido; perforación; abscesos; peritonitis. 


\section{Abstract}

Acute abdominal pain is a common illness in children. Acute appendicitis is the most common case in the emergency for abdominal surgery in children. It's about $80 \%$ of surgical emergencies. The diagnosis of acute appendicitis traditionally has been based on clinical criteria.

Most children with acute appendicitis have typical clinical features and undergo immediate surgery. However, 30-45\% have atypical clinical signs, and early diagnosis is difficult based on clinical findings alone, as children are unable to express their discomfort adequately and physical examinations may not be accurate and will delay surgery. The consequences of the delay may be intestinal perforation, abscesses and peritonitis.

In addition to the danger for the patient, can result in prolonged hospitalization and an increased risk of infertility in female patients. Thus, the persistent challenges are to establish the early diagnosis of atypical appendicitis, soon enough to prevent drilling and its consequences

Key words: Appendicitis; ultrasound; drilling; Abscesses; peritonitis. 
María Auxiliadora Calero-Zea; Blanca Elena Andrade-Burgos; Amando Sánchez-Alcívar; Julio Ramón Villacrés-

\section{Resumo}

A dor abdominal aguda é uma doença comum em crianças. A apendicite aguda é o caso mais comum na emergência para cirurgia abdominal em crianças. São cerca de $80 \%$ das emergências cirúrgicas. O diagnóstico de apendicite aguda tradicionalmente tem sido baseado em critérios clínicos.

A maioria das crianças com apendicite aguda apresentam características clínicas típicas e submetidas a cirurgia imediata. No entanto, 30-45\% apresentam sinais clínicos atípicos e o diagnóstico precoce é difícil, com base apenas nos achados clínicos, uma vez que as crianças não conseguem expressar seu desconforto de forma adequada e os exames físicos podem não ser precisos e atrasarão a cirurgia. As conseqüências do atraso podem ser perfuração intestinal, abscessos e peritonite.

Além do perigo para o paciente, pode resultar em hospitalização prolongada e aumento do risco de infertilidade em pacientes do sexo feminino. Assim, os desafios persistentes são estabelecer o diagnóstico precoce da apendicite atípica, o suficiente para evitar a perfuração e suas consequiências

Palavras chave: Apendicite; ultra-som; perfuração; abscessos; peritonite. 


\section{Métodos de diagnostico}

1.-RADIOGRAFIA SIMPLE DE ABDOMEN: Convencional, piedra angular para la evaluación de dolores abdominales agudos, pero la Radiología tiene un papel limitado en el diagnóstico de la apendicitis aguda.

El único hallazgo específico es el apendicolito, aunque esto ocurre solo en el 1- 15\% de los casos.

2.- ENEMA CON BARIO: Fue ampliamente usado en el diagnóstico de la Apendicitis aguda. Está basada en un apéndice que no llena, con un defecto extrínseco en la pared cecal.

Lo malo es que el apéndice no se llena en un $15 \%$ de niños sanos y otros tipos de enfermedades que pueden dar una imagen semejante.

Se conoce que la ultrasonografía es una prueba precisa para el diagnóstico de la apendicitis subaguda y es de gran ayuda para la apendicitis aguda junto con el laboratorio y la clínica.

La comprensión graduada es la técnica de ultrasonografía para el diagnóstico de la apendicitis aguda, y fue descrita por Puylaert en 1987.

Nuestro estudio se basa de ultrasonido como medio de ayudar en el diagnóstico de apendicitis aguda en niños, ver su sensibilidad, especificidad y exactitud de la comprensión graduada, y comparar sus resultados con los descubrimientos clínicos, quirúrgicos y anatomopalógicos. 
María Auxiliadora Calero-Zea; Blanca Elena Andrade-Burgos; Amando Sánchez-Alcívar; Julio Ramón VillacrésPastor

\section{Materiales y Métodos}

Se utilizaron para este estudio transductores de $5 \mathrm{MHZ}$ convexo y 7,5 MHZ. lineales en cortes transversales, longitudinales y oblicuos del cuadrante inferior derecho por medio de la técnica de comprensión graduada de Puylaert, un estudio del resto del abdomen se realizó si el apéndice no era visualizado.

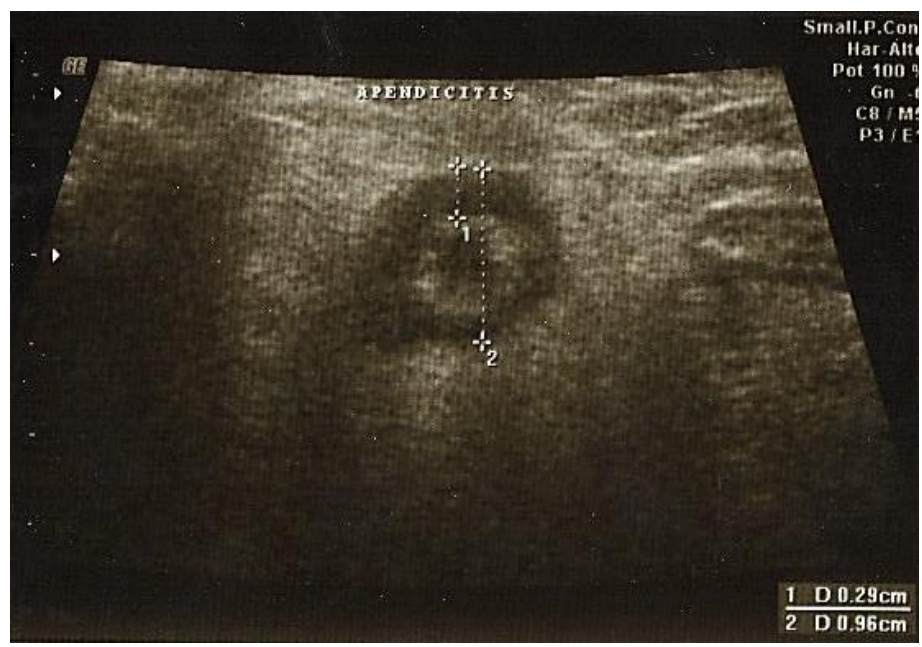

Fig 1.Imagen de donuts pared de 3mm diámetro de 9mm. Apendicitis

Se visualiza en corte transversal la submucosa y región asimétrica hipoecoica en la muscular que representa periapendicitis

El diámetro máximo de la sección de corte en el apéndice fue medido por un calibrador electrónico. La apendicitis era diagnosticada si era visualizada y no era comprimida, y si el diámetro era superior de $6 \mathrm{~mm}$. o si no un apendicolito o masa era notado. Si era de $6 \mathrm{~mm}$. o menos se puso en observación el paciente; con 7 mm. Ingresaba a quirófano. 


\section{Resultados.}

El estudio hecho en Aprofe desde enero - julio del 2013, se lograron reunir 27 casos, en los cuales se pudo realizar estudio ultrasonográfico; de estos, se diagnosticaron 18 apendicitis aguda y nueve pacientes fueron negativos para apendicitis. Los resultados de cirugía fueron: 19 intervenciones y 8 no intervenidos.

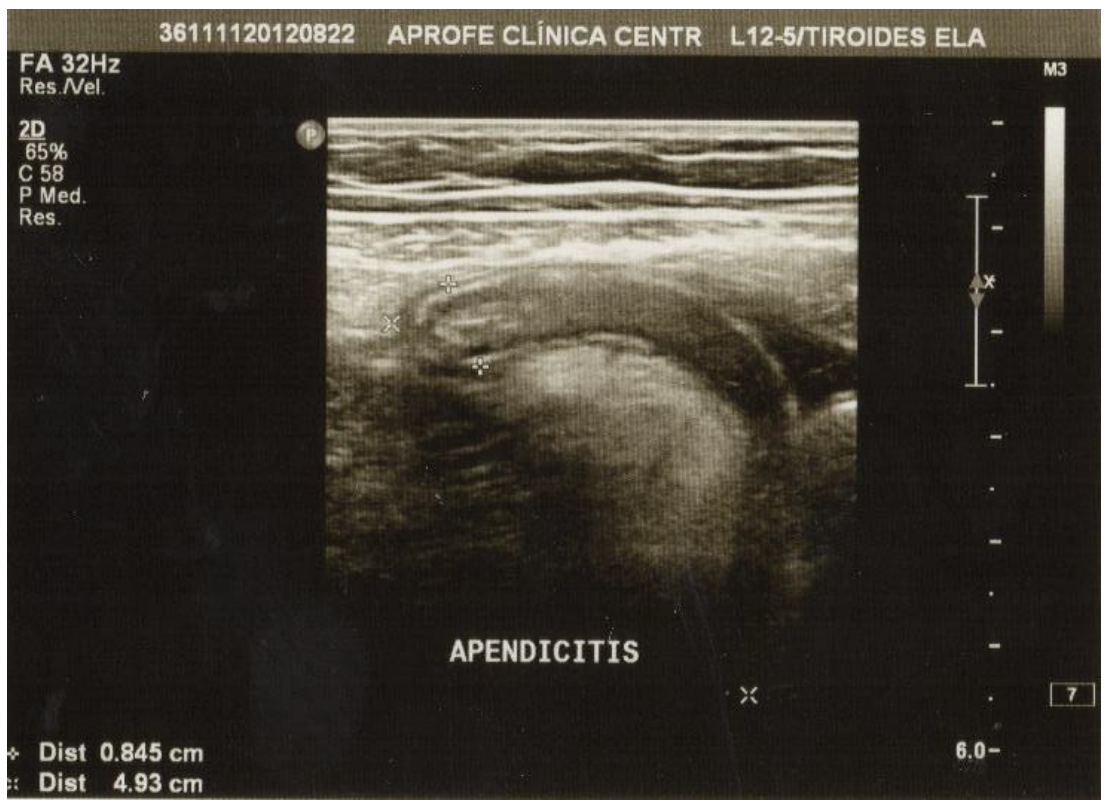

Fig 2.- Apéndice dilatada con pared de $4 \mathrm{~mm}$ y diámetro de $8,4 \mathrm{~mm}$

\section{Apendicitis}

Los resultados de patología fueron: 18 apendicitis agudas y una adenitis mesentérica + quistes de ovario derecho con pedículo torcido. De esto vemos que encontramos 1 falso positivo y un falso negativo. El falso negativo fue un score de menos de $6 \mathrm{~mm}$.

\section{Discusión.}

La apendicitis aguda en la infancia es de difícil diagnóstico. 
María Auxiliadora Calero-Zea; Blanca Elena Andrade-Burgos; Amando Sánchez-Alcívar; Julio Ramón VillacrésPastor

Clínicamente los niños están propensos a tener señales y por un período de tiempo más largo que en los adultos antes de hacer un diagnóstico correcto, por eso aumenta el riesgo de perforación.

La seguridad de descubrir apendicitis con exámenes de ultrasonografía en niños con una probabilidad baja o intermedia de apendicitis aguda basada en la clínica es alta.

En cambio el ultrasonido en niños con probabilidad alta de apendicitis aguda, sólo aumenta un poco la probabilidad clínica.

Larsen y colaboradores reportan que el valor del ultrasonido es cuestionable en pacientes con hallazgos fuerte de apendicitis aguda, mientras que, el desempeño del ultrasonido es garantizado y útil en pacientes con hallazgos clínicos ambíguos.

Estos resultados sugieren que la visualización del apéndice no puede ser tomada como único criterio de operación.

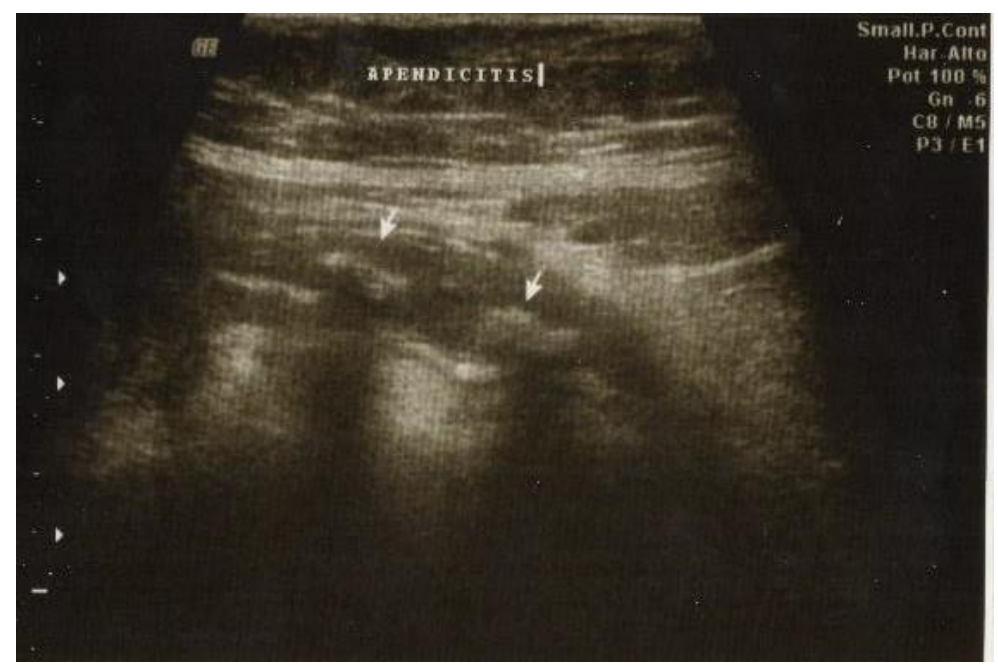

Fig 3.- Imágenes ecorefringentes dentro de la luz apendicular. 
Apendicolito se visuliza la submucosa distal. Apéndice no compresible

Debe ser combinada con hallazgos clínicos y de laboratorio El mayor limitante de la evaluación ultrasonografía es dificultad técnica y la comprensión en pacientes de dolor marcado.

\section{Conclusión.}

En conclusión la comprensión graduada en U.S., es útil para los diagnósticos de apendicitis en niños de bajo o intermedio riesgos clínicos de la enfermedad.

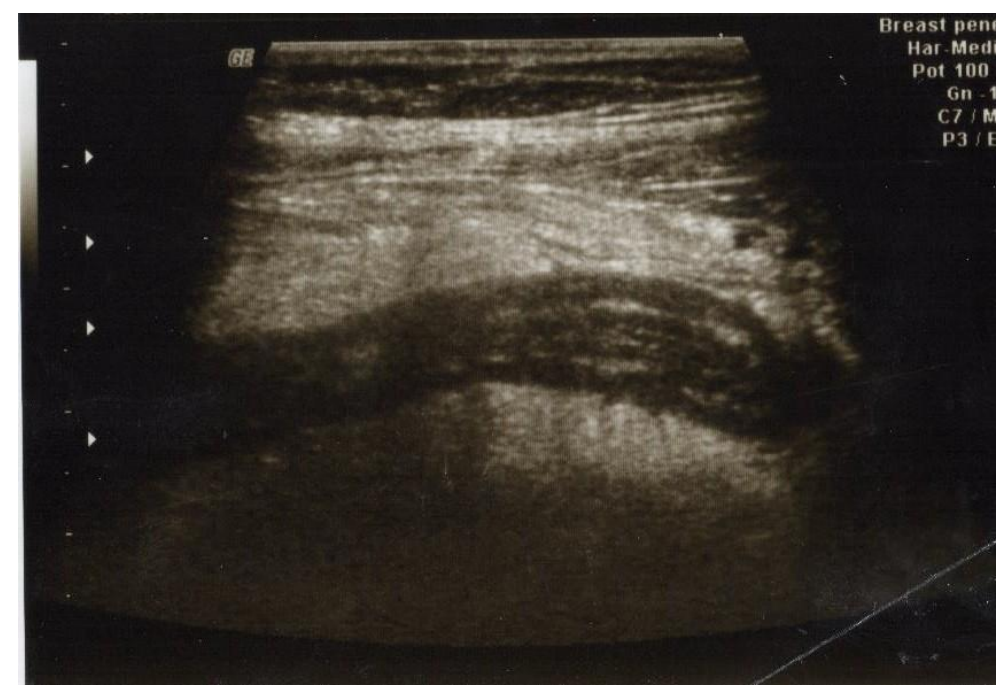

Fig 4 Corte longitudinal en el que se ve el fluído periapendicular, la submucosa ecogénica es

\section{identificada}

La realización de ultrasonido permite diagnósticos tempranos y establecer diferenciales.

En exámenes de alto riesgo, el ultrasonido, puede no ser necesario, y no contribuyente tanto como un examen clínico para la identificación de los pacientes con apendicitis. 


\section{Bibliografía}

1.-Borushock Karen F., R. Brooke Jeffrey Jr., et. Al Sonographic Diagnosis of Perforation in Patiens with Acute Appendicitis. AJR. 154: 275 - 278, February 1990.

2.- Garcia Merino F., Chavez Pesero F., y Col. Utilidad de la Ecografía en el Diagnostico de la Apendicitis Aguda en la infancia. Rev. Esp. Pediatrica: 45, 4 (278-182) 1989.

3.-Quillin Shawn P., Siegel Mrilyn J.,Coffin Cheryl M., Acute Appendicitis en children: Value of sonographic in Detecting Perforation, AJR 159, Dic, de 1992. (1265-1268).

4 .- R. Brooke Jeffrey Jr., Laing C., Faye, Lewis Frank R., Acutte Appendicitis: High Resolution Real Time U.S. Finding Radiology 1987 Abril 163: 11-14

5.- Rioux Michel. Sonographic Detection of the normal an abnormal Appendix AJR 158, Abril del 1992 (773-778)

6.- Yacoe M., Jeffrey B.: Sonography of apendicitis and diverticulitis. Radiologic Clinics of North America 1994, 32: 899-912.

7.- Jeffrey R., Laing F., Townsend R.: Acute appendicitis: sonographic criteria based on 250 cases.

Radiology 1988, 167: 327-329.

8.- Jeffrey R., Laing F., Lewis F.: Acute appendicitis: high-resolution real-time US findings. Radiology 1987, 163: 11-14. 